\title{
Protecting Against Hurricane Damage with Mathematics
}

\author{
Stefan Siegmund
}

The destruction left by hurricanes Harvey, Irma, and Maria can be measured in billions of dollars in damage and dozens of people injured or killed. We focus on using mathematics to prevent such losses due to wind.

As a sample for Notices readers, we now describe a model and device we developed, which helps to abate the risk of a complete loss of a mobile home during strong storms. The same principle could be applied to RVs, boats in dry storage facilities, planes, and larger buildings [1].

Imagine strong wind blowing against the side of a home. Similar to an airfoil of a plane the building acts as an obstacle to the passing airflow, which creates local pressure and suction at different parts of the roof (see Figure 1). In the worst case the uplift causes the whole roof to detach, often resulting in a total loss of the home.

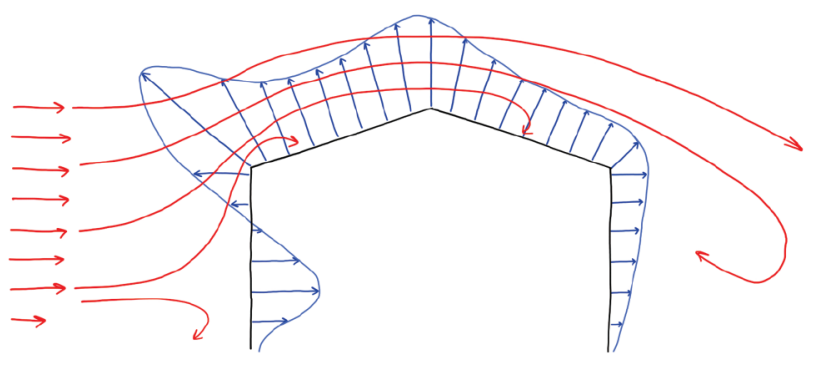

Figure 1: Wind (red) blowing against a building (black) creates pressure and suction/uplift (blue).

Mathematics can help to describe, analyze, and solve the problem of counteracting the uplift to hold the roof in place. As in Figure 2, netting panels, anchored in the

Stefan Siegmund is full professor for dynamics and control and director of the Center for Dynamics at TU Dresden, Germany. His email address is stefan.siegmund@tu-dresden.de.

For permission to reprint this article, please contact: reprint-permission@ams.org.

DOI: http://dx.doi.org/10.1090/noti1719 ground and connected to straps that cover the roof, redistribute the wind load and counteract the uplift.

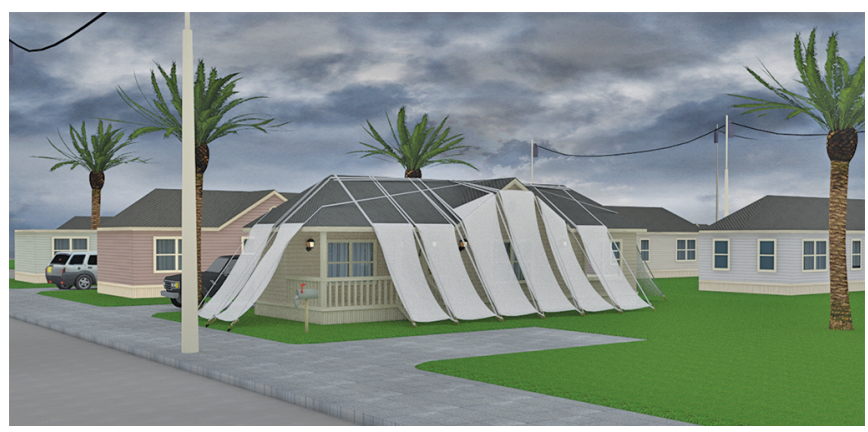

Figure 2: Netting panels with straps take some wind load and counteract the uplift.

Starting with a simple model, we assume that wind blows at right angles against the longer side of a building with roof inclination $\rho$ as in Figure 3.

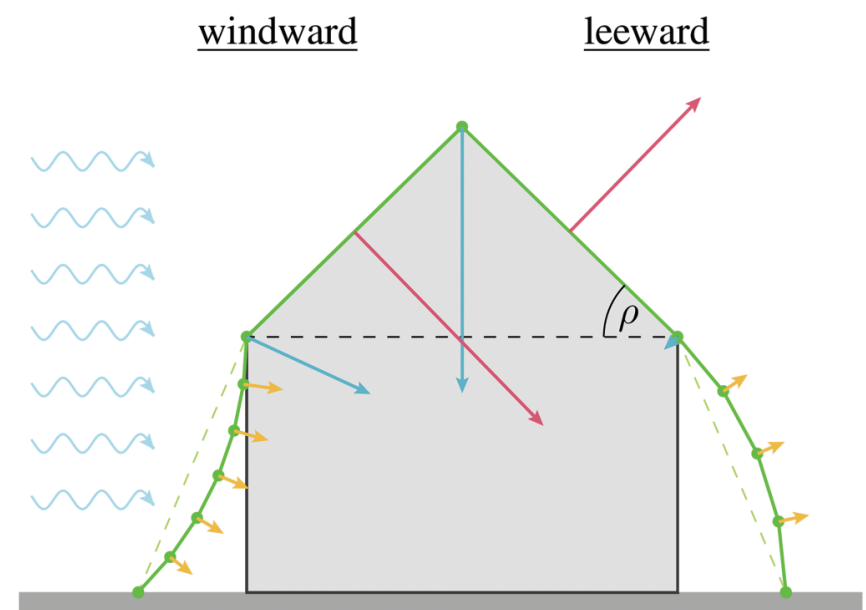

Figure 3: Wind (wiggled, blue arrows) creates pressure and uplift at roof (red force arrows). Nets and straps (green) take some wind load and counteract uplift (blue force arrows). 


\section{COMMUNICATION}

A cross section of the netting panel is modeled by a large number of connected short line segments. For the simplest case of only three segments, two pulleys at positions $\vec{X}_{1}$ and $\vec{X}_{2}$ are connected to springs with unit vectors $\vec{\xi}_{0}, \vec{\xi}_{1}, \vec{\xi}_{2}$, denoting the directions of the line segments. The forces $\overrightarrow{F_{1}}$ and $\overrightarrow{F_{2}}$ act on the corresponding pulleys, thereby deforming the system. The ends $\vec{X}_{0}$ and $\vec{X}_{3}$ are fixed (see Figure 4).

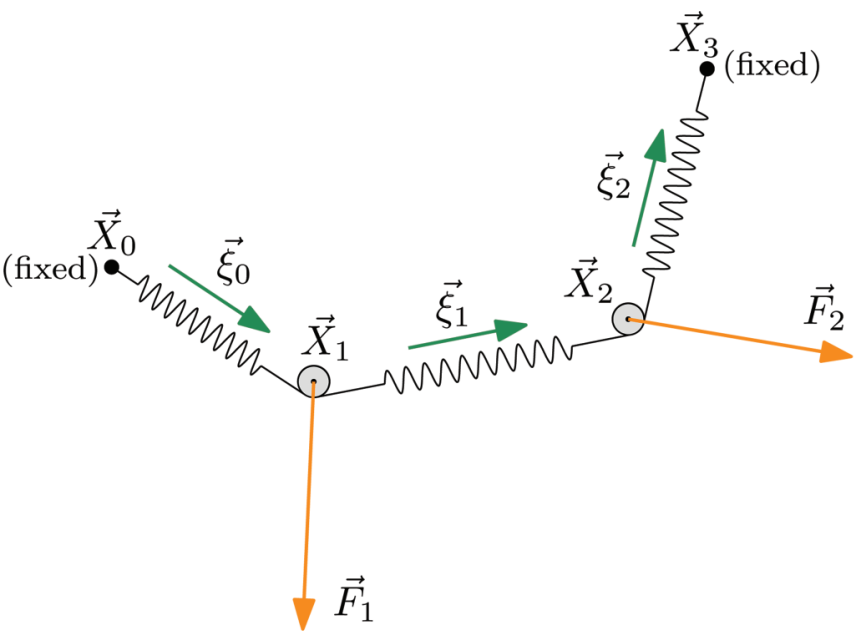

Figure 4: Cross section of netting panel modeled by three springs connected to pulleys and fixed ends.

In general, this is a dynamical problem. We focus on the equilibrium problem, i.e., the configuration the system relaxes to in the long run. In particular, we are interested in the equilibrium positions $\vec{X}_{1}$ and $\vec{X}_{2}$. A single spring of relaxed length $\ell$ with spring constant $\kappa$ stretched to length $\mathcal{L}$ is in the simplest case described by Hooke's law $s={ }_{\kappa}(\mathcal{L}-\ell)$. In equilibrium, the internal force $s$ along the

$\frac{\kappa}{\ell}$ cross section of the netting panel is constant and therefore $\overrightarrow{F_{1}}-s \vec{\xi}_{0}+s \vec{\xi}_{1}=0$ and $\vec{F}_{2}-s \vec{\xi}_{1}+s \vec{\xi}_{2}=0$. The slopes $\vec{\alpha}_{1}, \vec{\alpha}_{2}$ of the cross sections of the net at the positions $\vec{X}_{1}$ and $\vec{X}_{2}$ are defined by the slopes of the straight lines between their neighboring vertices; i.e., $\vec{\alpha}_{\kappa}=\arccos \left(\left(\vec{X}_{k+1}-\vec{X}_{\kappa-1}\right) \cdot \vec{e}_{1} / / \vec{X}_{\mathrm{k}+1^{-}}\right.$ $\left.\vec{X}_{\mathrm{k}-1} \mid\right)$, with the unit vector $\overrightarrow{\mathrm{e}}_{1}$ in horizontal wind direction. These angles are then used to compute effective wind forces on line segments. Altogether, the model relates those forces and the equilibrium positions of the vertices $\vec{X}_{\kappa}$ in a system of equations that can be solved numerically (see [2]). As a result, the forces within the straps and on the roof can be computed (see Figure 3, blue arrows).

How large is the uplift for a given wind speed? This depends on the roof inclination $\rho$ and varies along the roof. Engineers have computed the uplift; the German industry standard building codes DIN EN 1991-1-4 provide tables and formulas for position-dependent uplift in terms of $\rho$ (see Figure 3, red arrows). The mathematical model helps to determine the optimal netting size to counteract uplift over a wide range of wind speeds.

With the intention to help, my next steps were clear: build a prototype, test it in a wind tunnel, install it at a mobile home and test it in a real storm.
Over the last 10 years, TU Dresden and the German Klaus Tschira Foundation within their "Research for Society" program supported all steps. In October 2016, a trial installation north of Miami was hit by Hurricane Matthew with sustained wind speeds of over $100 \mathrm{mph}$. Whereas neighboring homes lost portions of their roofs or entire additions, the roof where the device was installed held firm.

\section{References}

[1] US PATENT 9,212,500, Device and method for wind loss prevention, Dec. 15, 2015.

[2] M.J. KÖRBER, S. SIEGMUND. Elastic model for a wind-protection membrane. J. Wind. Eng. Ind. Aerod. 165 (2017), 131-136.

\section{Image Credits}

Figure 1 by Stefan Siegmund.

Figure 2 by Martin Eggers and Stefan Siegmund.

Figures 3 \& 4 by Martin J. Körber and Stefan Siegmund.

Photo of Stefan Siegmund by Niels Eisfeld.

\section{ABOUT THE AUTHOR}

Stefan Siegmund works on dynamical systems theory. Recently he also focuses his time on research for society and work on geothermal power generation and seawater desalination.

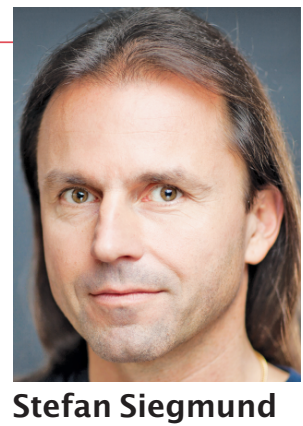

